\title{
Electron Emission from Laser-Irradiated Atomic Clusters ${ }^{1}$
}

\author{
Ulf Saalmann \\ Max Planck Institute for the Physics of Complex Systems, Nöthnitzer Straße 38, 01187 Dresden, Germany \\ e-mail: us@pks.mpg.de \\ Received September 29, 2008
}

\begin{abstract}
The mechanism of fast-electron emission from laser-irradiated clusters is studied by model calculations. Rescattering of electrons at the charged cluster is shown to be an important process. In contrast to rescattering at atoms, the structure, that is the size and strength, of the scattering potential determines final kinetic energies of the electrons. This becomes evident in a strong cluster-size dependence of the electron spectra.
\end{abstract}

PACS numbers: 33.80.Rv, 52.20.Fs, 36.40.Wa, 64.60.an

DOI: $10.1134 / \mathrm{S} 1054660 \mathrm{X} 0902008 \mathrm{X}$

\section{INTRODUCTION}

The response of atomic clusters on the irradiation by strong femtosecond laser pulses is, despite numerous experimental and theoretical investigations in the last 15 years (see recent reviews $[1,2]$ ), a field of active research. The interest is fostered by the observation of an extremely effective energy absorption by the clusters resulting in the emission of kiloelectronvolts-photons [3], highly charged ions $[4,5]$ or fast electrons $[6,7]$. While the basic absorption mechanism - the formation of a nano-plasma which can absorb resonantly energy if its eigenfrequency matches the laser frequency [8]is generally agreed by now [9-13], the mechanisms, e.g., of ion $[4,5,14,15]$ and electron $[6,7,16,17]$ acceleration are under current investigations. Here, we will focus on the emission of fast electron which turned out be determined by rescattering $[16,17]$ of electrons at the polarized/charged cluster.

Whereas the kinetic energies of ions are due to the Coulomb explosion of the charged cluster [18], the electron dynamics does not merely follow from their mutual repulsion but is strongly driven by the laser field. We will show below, that the measured energy spectra are due to an interplay of many-electron (laserheated nano-plasma inside the cluster) and single-electron (laser-assisted rescattering at the cluster) dynamics. We will discuss a novel mechanism [17], namely individual scattering of electrons at the cluster potential, and show that it is responsible for the observed kinetic energy spectra of electrons.

Fast electron generation has been discussed in terms of multiple scattering of electrons at different clusters in the gas [19], which should therefore depend on the cluster density. In this situation, scattering at the longrange Coulomb tail of the clusters dominates, whereas scattering inside the cluster is not crucial [19]. This does not apply for hot electrons which have just crossed the cluster border but are still in reach of the cluster's

\footnotetext{
${ }^{1}$ The article is published in the original.
}

Coulomb potential; they can be rescattered and the dominating laser-driven "inside scattering" will define their kinetic energy.

This situation is akin to the recollision in strongfield atomic physics [20]. There, however, the phase of the laser field at the electron's release time determines the final kinetic energy. A simple classical model [21], which captures the main physics, shows that the energy of direct electrons is not larger than $2 E_{\text {pond }}$ (the ponderomotive energy) and those which are backscattered [22] have energies not larger than $10 E_{\text {pond }}$. The structure of the scatterer does not play an important role; results are very similar for different atoms [23]. In contrast to that for extended scatterers [17], the structure of the scattering potential, in particular the charge state of the cluster, determines the electron energies.

\section{JELLIUM CLUSTERS IN STRONG LASER PULSES}

In order to keep the picture for the electron acceleration simple we study model clusters characterized by the total number of $N$ electrons, which are initially bound in a jellium sphere of homogenous charge density (with its integral being $N$ ) within a radius $R$. We neglect "creation" of new electrons through inner ionization [2] and expansion of the charged cluster, i.e., no bound electron is excited into the cluster and the cluster radius $R$ is fixed over the whole pulse. The first limitation is abolished by considering clusters with different electron densities. The latter one is not critical for the situations we are interested in. A quantitative estimation of the expansion dynamics is given in Appendix A.

The initial electron positions are determined by minimizing the total energy of $N$ particles interacting 

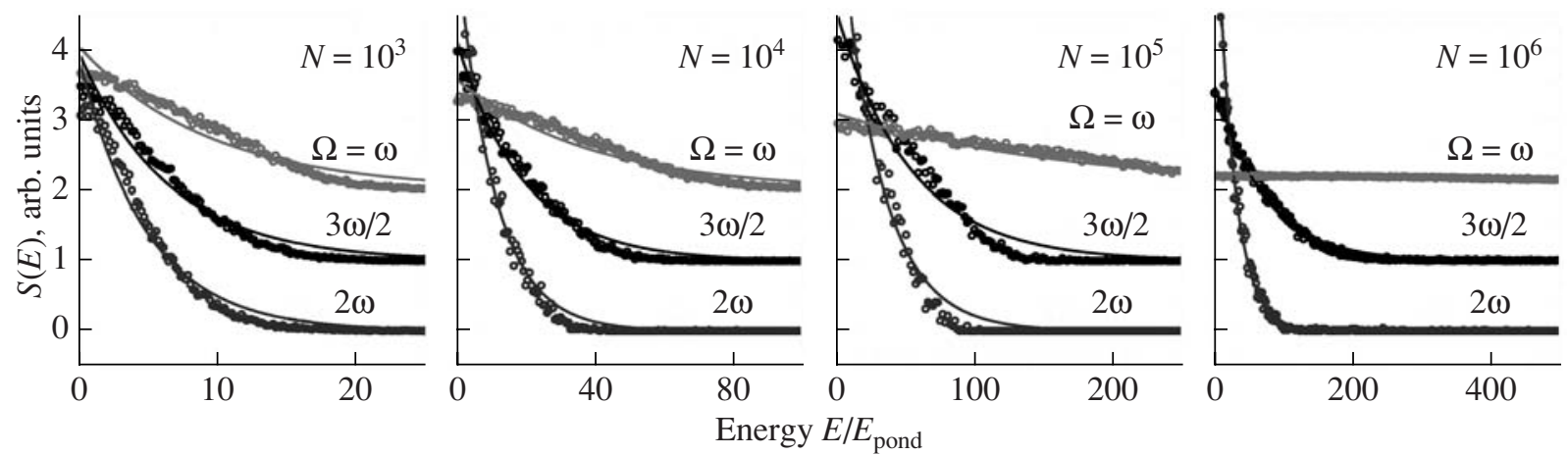

Fig. 1. Abundance of emitted electrons as a function of their kinetic energy $E$ for jellium clusters of different sizes $N=10^{3}, 10^{4}$, $10^{5}, 10^{6}$ (from left to right) and different eigenfrequencies $\Omega=2 \omega, 3 \omega / 2, \omega$, respectively. The pulse, cf. Eqs. (2) and (3), had a duration of $T=100 \mathrm{fs}$ and an intensity of $I=10^{14} \mathrm{~W} / \mathrm{cm}^{2}$. The spectra for the two lower eigenfrequencies are shifted upward for better visibility. Note the different scales for the energy $E$ which is given in terms of the ponderomotive energy $E_{\text {pond }}=5.67 \mathrm{eV}$. All spectra are fitted by exponential functions (solid lines).

through Coulomb repulsion in a jellium sphere with the potential

$$
V(r)= \begin{cases}\frac{N}{R} \frac{r^{2}-3 R^{2}}{2 R^{2}}, & r \leq R \\ -\frac{N}{r} & r \geq R .\end{cases}
$$

This is achieved by cooling the electronic system during the propagation after initialization in a random configuration. This procedure will most likely not lead to the global minimum which is, however, of no importance here since the system is strongly heated by the laser, whereby information about the exact initial configuration is quickly lost. The initialization and the response to the laser is obtained from molecular dynamics calculations solving classical equations of motion for the electrons. For particle numbers $N>10^{4}$ we have used the fast-multipole method (FMM) [24] in the implementation of Dachsel and Kabadshow [25]. Only thereby we could reach cluster sizes with as many as $N=10^{6}$ interacting electrons.

The driving force on the electrons is given by (linearly or circularly polarized) laser pulses characterized by their vector potentials

$$
\begin{gathered}
\mathscr{A}_{\operatorname{lin}}(t)=F(t) \mathbf{e}_{z} \cos (\omega t), \\
\mathscr{A}_{\text {circ }}(t)=F(t)\left[\mathbf{e}_{x} \cos (\omega t)+\mathbf{e}_{y} \sin (\omega t)\right] / \sqrt{2}
\end{gathered}
$$

with the envelope function for times $|t| \leq T$

$$
F(t)=\frac{\sqrt{I / I_{0}}}{\omega} \cos ^{2}\left(\frac{\pi}{2} \frac{t}{T}\right),
$$

where $I_{0}=3.51 \times 10^{16} \mathrm{~W} / \mathrm{cm}^{2}$. The center frequency was in all cases $\omega=1.59 \mathrm{eV}$ corresponding to a wavelength of $\lambda=780 \mathrm{~nm}$.

\section{KINETIC ENERGY OF RELEASED ELECTRONS}

We have performed systematic calculations of the ionization varying the cluster size $N$ over a wide range. Furthermore, we have studied different electron densities by choosing different cluster radii $R$ for a given $N$. Since they determine the eigenfrequency $\Omega$ of the spherical nano-plasma, their exact value is crucial if it is close to the laser frequency $\omega$ where absorption becomes resonant. Beside the resonant case $(\Omega=\omega)$ we have calculated higher eigenfrequencies $(\Omega>\omega)$ since this is the typical case for laser-cluster interaction where the eigenfrequency $\Omega$ change with time. At the rising edge of the pulse the charging leads to large $\Omega$ which is compensated later on by expansion of the cluster. This lowers $\Omega$ which eventually may become resonant with $\omega$ [12]. Since we omit cluster expansion here, we study the relevant cases separately.

We start by presenting the dependence of the electron spectra on the size of the clusters in Fig. 1. All spectra were obtained for pulses as specified in Eqs. (2) and (3) with a laser intensity of $I=10^{14} \mathrm{~W} / \mathrm{cm}^{2}$ and a pulse duration of $T=100 \mathrm{fs}$. The electrons were propagated long enough to ensure that the energy distributions $S(E)$ have reached their asymptotic shape. For $N=$ $10^{3}$ and $10^{4}$ we have averaged over 100 and 10 random orientations of the initial electron configuration, respectively, in order to improve the statistics. For $N=$ $10^{5}$ and $10^{6}$ we present single cluster results.

All spectra show a monotonic decrease for larger energies $E$. These decays can be approximated by an exponential function

$$
S_{E_{\text {kin }}}(E)=C \exp \left(-E / E_{\text {kin }}\right)
$$

with $E_{\text {kin }}$ a fitting constant and $C$ an irrelevant normalization factor. In discussing the electron energies we will refer to these fitting constants $E_{\text {kin }}$ simply as electron energies. They are presented in Fig. 2a. 


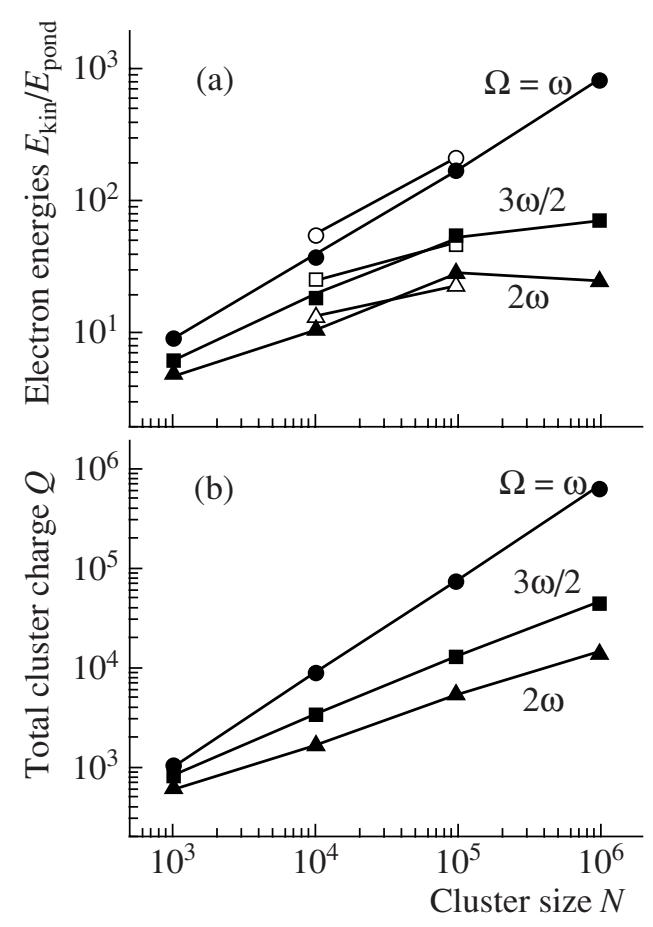

Fig. 2. Kinetic energy of electrons $E_{\text {kin }}$ (a) and total cluster charge $Q$ (b) after irradiation with a laser pulse as specified in Fig. 1. The values $E_{\text {kin }}$ are the fitting constants from Eq. (4) for the data presented in Fig. 1. Different symbols refer to different eigenfrequencies of the model clusters as specified. Open symbols (a) are from respective calculations far circularly polarized laser pulses. Note that both vertical axis are logarithmic.

Clearly, they depend strongly on the cluster size; note the different horizontal scale in Fig. 1 when going from left to right and the logarithmic vertical scale in Fig. 2a. The difference becomes most obvious for the resonant case $\Omega=\omega$. Here the electron energies are about two orders of magnitude larger $\left(E_{\mathrm{kin}} \approx 842 E_{\text {pond }}\right.$ for $N=10^{6}$ versus $E_{\text {kin }} \approx 9.4 E_{\text {pond }}$ for $N=10^{3}$ ) for the largest and smallest cluster sizes considered. Most notably are the large energies $E_{\text {kin }} \gg E_{\text {pond }}$ for the larger clusters which can only be explained by rescattering at the strongly charged cluster as discussed below. Whereas the laser intensity $I$ is the same in all cases, the cluster charges $Q$ as observed at the end of the pulse differ strongly as can be seen in Fig. 2b. The systematically larger charges $Q$ for smaller eigenfrequencies $\Omega$ are due to the effective collisionless heating of the cluster's nano-plasma close to the resonance $[8,12]$.

Obviously, there is a correlation between the electron energies $E_{\text {kin }}$ and the cluster charges $Q$. In order to quantify this effect we have developed a simple rescattering model for extended systems [17]. The idea was borrowed from the three-step model [20] of laser-atom interaction. There, the electron, after release from the atom and acceleration back towards the atoms, can be elastically back-scattered at the atom. If this happens at

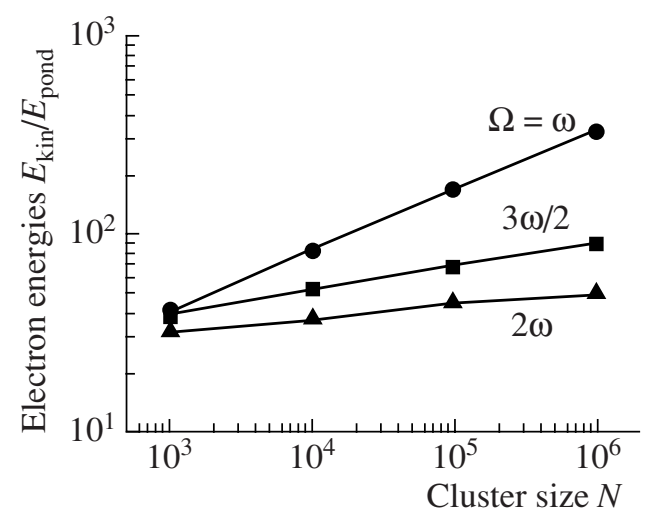

Fig. 3. Kinetic energy of electrons $E_{\text {kin }}$ according to the rescattering model, Eq. (5), for the clusters presented in Figs. 1 and 2 .

the right phase of the electric field [22] the final kinetic energy will be considerably larger than for direct electrons, i.e., those electrons which are not rescattered. There are two main differences when going from atoms to clusters: Firstly, the electron dynamics in clusters is not phase-locked with the laser. Rather a plasma is formed in the cluster and electrons may undergo multiple collisions before they may end up in the cluster's Coulomb tail from where they may be driven back to the cluster potential. Secondly, the scattering process itself differs by the finite extension of the scatterer. This is discussed in detail in Appendix B. It gives the maximal velocity an electron can acquire by passing an extended potential in the presence of an oscillating field, cf. Eq. (B9). The corresponding energy gain reads

$$
\Delta E=4 \sqrt{E_{\text {pond }} V} .
$$

This equation shows clearly the dependence of the electron energies on the scattering potential in agreement with the observation in Fig. 2. It is qualitatively different from the rescattering at atoms where the structure of the scatterer is of minor importance [23]. Using the ponderomotive energy of the peak intensity and the charges $Q$ as obtained from the calculation, cf. Fig. 2b, we can estimate $V$ and thus the final kinetic energies according to Eq. (5). The corresponding values are presented in Fig. 3. The overall trend for the kinetic energies is quantitatively reproduced. Deviations are mainly for small clusters $\left(N=10^{3}\right)$ where the model overestimates the energies which could be due to the fact that ionization occurs early in the pulse, i.e., at field strengths smaller than the peak field strength.

It is known that increasing the ellipticity of the polarization reduces the backscattering signal for atoms [23]. The reason is that the electron does not hit that atom (or ion) since the other laser component drives it away from the rescattering axis. This is different in the case of clusters where plasma heating continuously brings electrons into the cluster's Coulomb tail from where rescattering can occur. Thus one would expect 

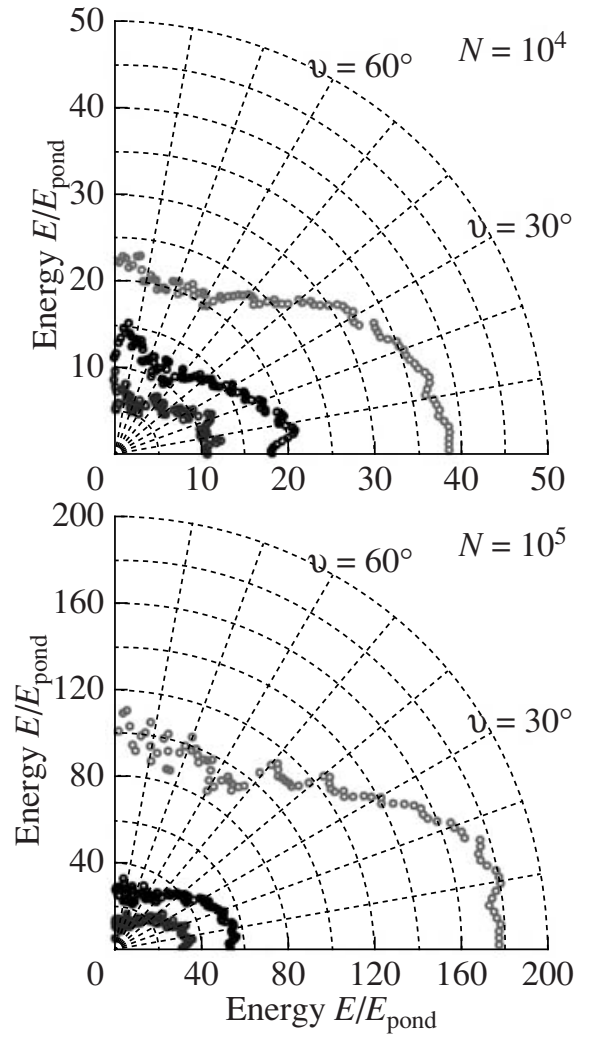

Fig. 4. Angular distributions for linearly polarized laser pulses with $I=10^{14} \mathrm{~W} / \mathrm{cm}^{2}$ and $T=100 \mathrm{fs}$. Shown are the fitting constants $E_{\text {kin }}$ as a function of the polar angle $\theta$, the angle between laser polarization axis and direction of observation.

similar results for the electron energies for circular polarization. Indeed, as can be seen by the open symbols in Fig. 3a, results for circularly polarized even quantitatively agree with those for linearly polarized light.

Energy distributions are often attributed to the thermal energy of electrons in the cluster. Our calculations show that values for the final kinetic energy and typical kinetic energies of plasma electrons ("temperature") differ considerably. We will not present absolute values of this temperature for two reasons. First of all it changes during the pulse. Secondly, the velocity distribution of the plasma electrons does not have a Maxwell-Boltzmann shape due to the strong driving and electrons that are rescattered at the cluster. Plasma electron energies at the peak of the pulse ranges from about $100 \mathrm{eV}$ for clusters with $N=10^{3}$ electrons to about $400 \mathrm{eV}$ for clusters with $N=10^{6}$ electrons. In contrast to that, final kinetic energies change much more, namely from about $50 \mathrm{eV}$ to about $5 \mathrm{keV}$, cf. Fig. $2 \mathrm{a}$ at $\Omega=\omega$, for these clusters.

Another indicator for non-evaporative release of electrons are angular distributions of electrons. Whereas Fig. 1 summarizes over all electrons, Fig. 4 shows the fitting constants as a function of the polar angle $\vartheta$, the angle between laser polarization axis and direction of observation. We present here results for $N=10^{4}$ and $10^{5}$. Independent of the cluster's eigenfrequency $\Omega$ and thus the absolute values of the electron energies $E_{\text {kin }}$, the fastest electrons are ejected along the laser polarization direction, i.e. for $\vartheta=0^{\circ}$. The fitting constants for parallel ejection $\left(\vartheta=0^{\circ}\right)$ are about two times larger than those for perpendicular ejection $(\vartheta=$ $90^{\circ}$ ). Furthermore, our data do not only show that the electrons ejected along the polarization are faster but also that the absolute ejection is stronger along the laser polarization. The preferred ejection along the polarization axis has been observed experimentally for large xenon clusters [7] as well as for small silver clusters [16].

\section{CONCLUSIONS}

We have shown by model calculation with jellium clusters that the emission energy of electrons strongly depends on the cluster they are emitted from. For identical driving fields electrons are considerably faster if the cluster is more strongly charged. This is due to rescattering of electrons at the cluster's potential in the presence of an oscillating laser field. A generalization of the simple rescattering model for atoms explains the dependence of the kinetic energies on this cluster potential. Angular distributions indicate that the electron emission is preferred in the polarization direction with faster electrons than perpendicular to it. This rules out evaporation from the hot nano-plasma in the cluster as the mechanisms for fast electron production.

\section{APPENDIX A}

\section{CLUSTER EXPANSION}

We estimate the relevance of the cluster expansion by looking at the hypothetical dynamics of an ion at the cluster surface. The electric field at the surface of a cluster with a time-dependent charge $Q(t)$ is

$$
\mathscr{E}(t)=\frac{Q(t)}{R^{2}(t)}<\frac{Q(t)}{R_{\text {init }}^{2}},
$$

the latter being an upper limit. Assuming that the cluster charging can be approximated by

$$
Q(t)=\frac{Q_{\text {final }}}{1+\exp \left(-t / T_{Q}\right)},
$$

with $T_{Q}$ the "charging duration." We obtain an upper limit for the radius from integrating Newton's equation 


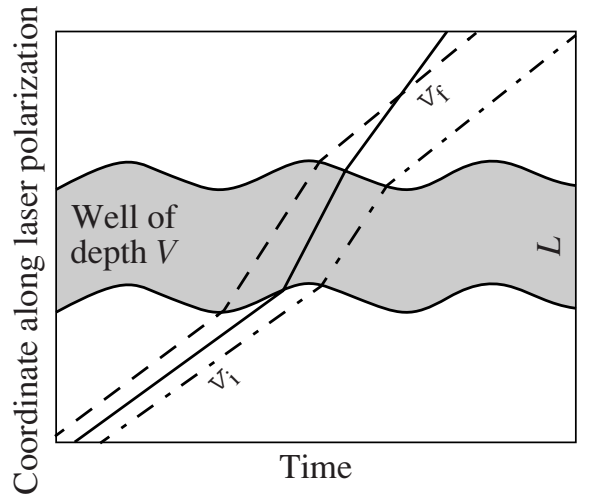

Fig. 5. Three electron trajectories (straight lines) passing an oscillating finite well (gray-shaded area). The initial velocity $v_{\mathrm{i}}$ is the same in all three cases. Entering and leaving the well potential at different times/phases of the oscillation leads to different final velocities $V_{\mathrm{f}}$. The strength of the kinks at the borders, as given by Eq. (B.2), depend beside on the potential depths also on the respective velocities of the potential at the crossing times.

$m \ddot{R}(t)=q \mathscr{E}(t)$ for an atom with mass $m$ and charge $q$

$$
\begin{aligned}
& R(t)<R_{\text {init }}+\frac{q}{m} \int d t^{t} \int d t^{\prime \prime} \mathscr{E}\left(t^{\prime \prime}\right) \\
& =R_{\text {init }}-\frac{q}{m} \operatorname{Li}_{2}\left(-e^{t / T_{Q}}\right) \frac{Q_{\text {final }}}{R_{\text {init }}^{2}} T_{Q}^{2}
\end{aligned}
$$

with the poly-logarithm function $\mathrm{Li}_{2}$. At the end of the charging, i.e., at time $t=T_{Q}$, one gets $\operatorname{Li}_{2}(-\mathrm{e}) \approx-1.806$ and thus

$$
\Delta R:=R\left(T_{Q}\right)-R_{\text {init }}<\frac{2 q}{m} \frac{Q_{\text {final }}}{R_{\text {init }}^{2}} T_{Q}^{2} .
$$

This gives an upper limit for the expansion $\Delta R$. Absolute values for some clusters considered above are give in table. Even for the most extreme case the expansion would not be larger than $10 \%$ during the laser-cluster interaction time.

Compilation of radial changes $\Delta R$ giving an upper limit of expansion during the charging time $T_{Q} \approx 50 \mathrm{fs}$ according to Eq. (A.4). For $m$ we have used the atomic mass of xenon and $q=1$. Estimating the atomic charge $q$ from $Q$ using the xenon density would give even smaller values $q$ and thus smaller changes $\Delta R$ of the radii

\begin{tabular}{c|c|c|c}
\hline Cluster $(N, \Omega)$ & $R_{\text {init }}$ & $Q_{\text {final }}$ & $\Delta R$ \\
\hline$\left(10^{3}, 2 \omega\right)$ & $35 \AA$ & 583 & $1.8 \AA$ \\
$\left(10^{3}, \omega\right)$ & $35 \AA$ & 945 & $3.5 \AA$ \\
$\left(10^{6}, 2 \omega\right)$ & $351 \AA$ & $1.34 \times 10^{4}$ & $0.5 \AA$ \\
$\left(10^{6}, \omega\right)$ & $351 \AA$ & $6.27 \times 10^{5}$ & $26 \AA$ \\
\hline
\end{tabular}

APPENDIX B RESCATTERING AT A FINITE WELL POTENTIAL

In order to write down the rescattering analytically we consider a finite well potential of width $L$ and depth $V$. For comparison with the numerical data in Section 3 we set later $L=2 R$ and depth $V=(3 Q) /(2 R)$, see Eq. (1). We use the Kramers-Henneberger frame, where the potential oscillates according to the motion of a free electron in an oscillating field, depicted as gray-shaded area in Fig. 5. Considering that the potential is constant outside one gets the final electron velocity directly without any oscillating part as in the frame using the length form [17]. We assume a sinusoidal form of the potential's velocity

$$
V_{\text {well }}(t)=\mathscr{A}_{0} \cos (\omega t) \text {. }
$$

The electron's velocity is constant apart from the jumps at the cluster border, which show up as kinks in the trajectories in Fig. 5. We will use the symbols $v_{i}, v_{t}$, and $v_{\mathrm{f}}$ for its initial, transient and final velocity, respectively. These three velocities are connected by formulas guaranteeing energy conservation (in the moving frame) at the times $t_{1}$ and $t_{2}$, when the electron enters or leaves the potential well

$$
\begin{aligned}
& \left(V_{\mathrm{t}}-v_{1}\right)^{2}=\left(v_{\mathrm{i}}-v_{1}\right)^{2}+2 V, \\
& \left(v_{\mathrm{f}}-v_{2}\right)^{2}=\left(v_{\mathrm{t}}-v_{2}\right)^{2}-2 V .
\end{aligned}
$$

with $v_{1}=V_{\text {well }}\left(t_{1}\right)$ and $v_{2}=V_{\text {well }}\left(t_{2}\right)$ the well's velocity at the crossing times.

Since for the case we are interested in, the time difference $t_{2}-t_{1}$ is smaller than one laser period $2 \pi / \omega$ we will use below the phases $\varphi_{1}:=\omega t_{1}$ and $\varphi_{2}:=\omega t_{2}$ to characterize the crossings of the potential borders. The final velocity reads

$$
v_{\mathrm{f}}=v_{2}+\sqrt{\left[v_{1}-v_{2}+\sqrt{\left[v_{\mathrm{i}}-v_{1}\right]^{2}+2 V}\right]^{2}-2 V} \text {, }
$$

which is of course equivalent to the expression derived for the electric field in length form [17].

The phases $\varphi_{1}$ and $\varphi_{2}$, thus the velocities $v_{1}$ and $v_{2}$, can not be chosen arbitrary but are connected through the external parameter $v_{i}, L, V$, and $\mathscr{A}_{0}$. One can calculate the width $\tilde{L}$, the potential should have, for a given combination of $\varphi_{1}$ and $\varphi_{2}$ from

$$
v_{\mathrm{t}}\left(t_{2}-t_{1}\right)=\left(x\left(t_{2}\right)+\tilde{L} / 2\right)-\left(x\left(t_{1}\right)-\tilde{L} / 2\right)
$$

and obtains the equation

$$
\begin{gathered}
\tilde{L} \omega=\left[v_{1}+\sqrt{\left[v_{\mathrm{i}}-v_{1}\right]^{2}+2 V}\right]\left(\varphi_{2}-\varphi_{1}\right) \\
+\mathscr{A}_{0}\left(\cos \varphi_{2}-\cos \varphi_{1}\right) .
\end{gathered}
$$

For a given $L$, Eq. (B.5) is an implicit equation connecting $\varphi_{1}$ and $\varphi_{2}$. 
In order to find the values of $v_{1}$ and $v_{2}$ which makes $v_{\mathrm{t}}$ and $V_{\mathrm{f}}$ extremal, we look at the derivations with respect to these velocities

$$
\begin{aligned}
& \frac{d}{d V_{1}} V_{\mathrm{t}}=1-\left(V_{\mathrm{i}}-v_{1}\right) / \sqrt{\left(V_{\mathrm{i}}-v_{1}\right)^{2}+2 V}, \\
& \frac{d}{d v_{2}} V_{\mathrm{f}}=1-\left(v_{\mathrm{t}}-v_{2}\right) / \sqrt{\left(v_{\mathrm{t}}-v_{2}\right)^{2}+2 V} .
\end{aligned}
$$

Limiting $V_{\mathrm{i}} \geq \mathscr{A}_{0} \geq V_{1}$ and assuming that the electron does not get trapped $\left(V_{t}-V_{2}\right)^{2}>2 V$, the derivatives for $V_{\mathrm{t}}$ and $V_{\mathrm{f}}$ in Eqs. (B.6), are always positive or negative, respectively. Thus the optimal crossings are at $v_{1}=+\mathscr{A}_{0}$ and $v_{2}=-\mathscr{A}_{0}$. With other words, for maximal $v_{\mathrm{t}}$ and $v_{\mathrm{f}}$ the potential should move along with electron for the step down and against it for the step up, cf. Fig. 5. The maximal final velocity reads

$$
+\sqrt{\left[2 \mathscr{A}_{0}+\sqrt{\left[v_{\mathrm{i}}-\mathscr{A}_{0}\right]^{2}+2 V}\right]^{2}-2 V}
$$

and is achieved only if the potential width fulfills the following equation

$$
\begin{aligned}
\tilde{L}^{\max } \omega=[ & \left.\mathscr{A}_{0}+\sqrt{\left[v_{\mathrm{i}}-\mathscr{A}_{0}\right]^{2}+2 V}\right]\left(\varphi_{2}-\varphi_{1}\right) \\
& +\mathscr{A}_{0}\left(\cos \varphi_{2}-\cos \varphi_{1}\right) .
\end{aligned}
$$

Both expressions (B.7) and (B.8) simplify if one assumes that the potential's transient velocity is much larger than the initial and the quiver velocity $\sqrt{2 V} \gg V_{\mathrm{i}}$ and $\sqrt{2 V} \gg \mathscr{A}_{0}$. Both assumptions are fulfilled in the calculations presented above. Finally we get

$$
\begin{aligned}
V_{\mathrm{f}}^{\max } & =2 \sqrt{\mathscr{A}_{0} \sqrt{2 V}}, \\
\tilde{L}^{\max } & =\sqrt{2 V} \pi / \omega .
\end{aligned}
$$

The first equation gives the optimal value for the velocity that can be acquired, the second one is a requirement which should be fulfilled in order to acquire it.

\section{ACKNOWLEDGMENTS}

Many helpful discussions with Christian Gnodtke regarding the propagation with and without FMM and with Jan Michael Rost about the rescattering model, a critical reading of the manuscript by Inout Georgescu and support regarding the implementation of the FMM by Ivo Kabadshow are gratefully acknowledged.

\section{REFERENCES}

1. V. P. Krainov and M. B. Smirnov, Phys. Rep. 370, 237 (2002).

2. U. Saalmann, C. Siedschlag, and J. M. Rost, J. Phys. B 39, R39 (2006).

3. A. McPherson, B. D. Thompson, A. B. Borisov, K. Boyer, and C. K. Rhodes, Nature 370, 631 (1994).

4. T. Ditmire, J. W. G. Tisch, E. Springate, M. B. Mason, N. Hay, R. A. Smith, J. Marangos, and M. H. R. Hutchinson, Nature 386, 54 (1997)

5. M. Lezius, S.Dobosz, D. Normand, and M. Schmidt, Phys. Rev. Lett. 80, 261 (1998)

6. E. Springate, S. A. Aseyev, S. Zamith, and M. J. J. Vrakking, Phys. Rev. A 68, 053201 (2003)

7. V. Kumarappan, M. Krishnamurthy, and D. Mathur, Phys. Rev. A 67, 043204 (2003)

8. T. Ditmire, T. Donnelly, A. M. Rubenchik, R. W. Falcone, and M. D. Perry, Phys. Rev. A 53, 3379 (1996)

9. L. Köller, M. Schumacher, J. Köhn, S. Teuber, J. Tiggesbäumker, and K.-H. Meiwes-Broer, Phys. Rev. Lett. 82, 3783 (1999)

10. J. Zweibach, T. Ditmire, and M. D. Perry, Phys. Rev. A 59, R3166 (1999)

11. I. Last and J. Jortner, Phys. Rev. A 60, 2215 (1999)

12. U. Saalmann and J. M. Rost, Phys. Rev. Lett. 91, 223401 (2003)

13. U. Saalmann, J. Mod. Opt. 53, 173 (2006)

14. V. Kumarappan, M. Krishnamurthy, and D. Mathur, Phys. Rev. Lett. 87, 085005 (2001)

15. M. Krishnamurthy, D. Mathur, and V. Kumarappan, Phys. Rev. A 69, 033202 (2004)

16. T. Fennel, T. Döppner, J. Passig, C. Schaal, J. Tiggesbäumker, and K.-H. Meiwes-Broer, Phys. Rev. Lett. 98, 143401 (2007).

17. U. Saalmann and J. M. Rost, Phys. Rev. Lett. 100, 133006 (2008)

18. M. R. Islam, U. Saalmann, and J. M. Rost, Phys. Rev. A 73, R041201 (2006)

19. M. B. Smirnov and V. P. Krainov, Phys. Plasma 10, 443 (2003)

20. P. B. Corkum, Phys. Rev. Lett. 71, 1994 (1993)

21. P. B. Corkum, N. H. Burnett, and F. Brunel, Phys. Rev. Lett. 62, 1259 (1989)

22. G. G. Paulus, W. Becker, W. Nicklich, and H. Walther, J. PHys. B 27, L703 (1994)

23. G. G. Paulus, F. Zacher, H. Walther, A. Lohr, W. Becker, and M. Kleber, Phys. Rev. Lett. 80, 484 (1998)

24. L. Greengard and V. Rokhlin, J. Comput. Phys. 73, 325 (1987)

25. H. Dachsel and I. Kabadshow, http://www.fz-juelich.de/ jsc/fmm (2008) 